Proceedings of the Edinburgh Mathematical Society (2007) 50, 597-603 (C)

DOI:10.1017/S0013091505001380 Printed in the United Kingdom

\title{
MULTIPLE SOLUTIONS FOR A QUASILINEAR ELLIPTIC VARIATIONAL SYSTEM ON STRIP-LIKE DOMAINS
}

\author{
F. CAMMAROTO*, A. CHINNÍ AND B. DI BELLA \\ Department of Mathematics, University of Messina, Salita Sperone 31, \\ 98166 Sant'Agata-Messina, Italy (bdibella@unime.it)
}

(Received 4 October 2005)

Abstract We consider the quasilinear elliptic variational system

$$
\begin{aligned}
-\Delta_{p} u & =\lambda F_{u}(x, u, v)+\mu H_{u}(x, u, v) & & \text { in } \Omega, \\
-\Delta_{q} v & =\lambda F_{v}(x, u, v)+\mu H_{v}(x, u, v) & & \text { in } \Omega, \\
u & =v=0 & & \text { on } \partial \Omega,
\end{aligned}
$$

where $\Omega$ is a strip-like domain and $\lambda$ and $\mu$ are positive parameters. Using a recent two-local-minima theorem and the principle of symmetric criticality, existence and multiplicity are proved under suitable conditions on $F$.

Keywords: Strip-like domain; eigenvalue problem; elliptic systems

2000 Mathematics subject classification: Primary 35A15, 35J65

Secondary $35 \mathrm{P} 30$

\section{Introduction}

Very recently, in [1] Kristály studied the eigenvalue problem

$$
\left.\begin{array}{rlrl}
-\Delta_{p} u & =\lambda F_{u}(x, u, v) & & \text { in } \Omega, \\
-\Delta_{q} v & =\lambda F_{v}(x, u, v) & & \text { in } \Omega, \\
u & =v=0 & & \text { on } \partial \Omega,
\end{array}\right\}
$$

where $\lambda>0$ is a parameter and $\Omega$ is a strip-like domain in $\mathbb{R}^{N}$, i.e. $\Omega=\omega \times \mathbb{R}^{l}, \omega$ being a bounded open subset of $\mathbb{R}^{m}$ with smooth boundary, $m \geqslant 1, l \geqslant 2,1<p, q<N=m+l$, $F \in C^{0}\left(\Omega \times \mathbb{R}^{2}, \mathbb{R}\right)$, and $\Delta_{\alpha} w=\operatorname{div}\left(|\nabla w|^{\alpha-2} \nabla w\right)$. Here, $F_{z}$ denotes the partial derivative of $F$ with respect to variable $z$. He applies a critical point result (see [5]) in order to obtain the existence of an open interval $\Lambda \subset(0,+\infty)$ such that, for every $\lambda \in \Lambda$, the system $S_{\lambda}$

* Because of a surprising coincidence of names within our department, we have to point out that the author was born on 4 August 1968. 
has at least two distinct non-trivial solutions. Also, he assumes that the nonlinear term $F$ is sub- $p, q$-linear; that is,

$\left(1_{F}\right) \lim _{u, v \rightarrow 0} \frac{F_{u}(x, u, v)}{|u|^{p-1}}=\lim _{u, v \rightarrow 0} \frac{F_{v}(x, u, v)}{|v|^{q-1}}=0$, uniformly w.r.t. $x \in \Omega$.

Inspired by [1] , we prove two multiplicity theorems, which extend the results contained in $[\mathbf{1}]$, for the system

$$
\left.\begin{array}{rlrl}
-\Delta_{p} u & =\lambda F_{u}(x, u, v)+\mu H_{u}(x, u, v) & & \text { in } \Omega, \\
-\Delta_{q} v & =\lambda F_{v}(x, u, v)+\mu H_{v}(x, u, v) & & \text { in } \Omega, \\
u & =v=0 & & \text { on } \partial \Omega
\end{array}\right\}
$$

where $\mu$ is a positive parameter. Our approach is based on a recent result of Ricceri $[\mathbf{6}$, Theorem 4]; in a convenient form for our purposes it can be read as follows.

Theorem 1.1 (Ricceri). Let $X$ be a reflexive real Banach space, let $I \subseteq \mathbb{R}$ be an interval, and let $\Psi: X \times I \rightarrow \mathbb{R}$ be a function such that $\Psi(x, \cdot)$ is concave in $I$ for all $x \in X$, while $\Psi(\cdot, \lambda)$ is continuous, coercive and sequentially weakly lower semicontinuous in $X$ for all $\lambda \in I$. Further, assume that

$$
\sup _{\lambda \in I} \inf _{x \in X} \Psi(x, \lambda)<\inf _{x \in X} \sup _{\lambda \in I} \Psi(x, \lambda) .
$$

Then, for each $\rho>\sup _{I} \inf _{X} \Psi(x, \lambda)$ there exists a non-empty open set $A \subseteq I$ with the following property: for every $\lambda \in A$ and every sequentially weakly lower semicontinuous functional $\Phi: X \rightarrow \mathbb{R}$, there exists $\delta>0$ such that, for each $\mu \in] 0, \delta[$, the functional $\Psi(\cdot, \lambda)+\mu \Phi(\cdot)$ has at least two local minima lying in the set $\{x \in X: \Psi(x, \lambda)<\rho\}$.

In the present paper, the function $F$ is assumed to be a $C^{0}\left(\Omega \times \mathbb{R}^{2}, \mathbb{R}\right)$ function such that

$\left(2_{F}\right) F$ is axially symmetric in the first variable; that is,

$$
F\left(\left(x_{1}, x_{2}\right), s, t\right)=F\left(\left(x_{1}, g x_{2}\right), s, t\right) \quad \text { for all } x_{1} \in \omega, x_{2} \in \mathbb{R}^{l}, g \in O(l),(s, t) \in \mathbb{R}^{2} ;
$$

$\left(3_{F}\right)(s, t) \rightarrow F(x, s, t)$ is of class $C^{1}$ and $F(x, 0,0)=0$ for all $x \in \Omega$.

Moreover, let $\alpha^{*}=N \alpha /(N-\alpha), \alpha \in\{p, q\}$, be the critical Sobolev exponent and we assume that

$\left(4_{F}\right)$ there exist $\varepsilon>0$, and $\left.r \in\right] p, p^{*}[, s \in] q, q^{*}[$, with $p s=q r$, such that

$$
\begin{aligned}
& \left|F_{u}(x, u, v)\right| \leqslant \varepsilon\left(|u|^{p-1}+|v|^{(p-1) q / p}+|u|^{r-1}\right), \\
& \left|F_{v}(x, u, v)\right| \leqslant \varepsilon\left(|v|^{q-1}+|u|^{(q-1) p / q}+|v|^{s-1}\right)
\end{aligned}
$$

for each $x \in \Omega$ and $(u, v) \in \mathbb{R}^{2}$. 
Throughout this paper, the norm on $W_{0}^{1, \alpha}(\Omega)$ is defined by

$$
\|u\|_{\alpha}=\left(\int_{\Omega}|\nabla u|^{\alpha}\right)^{1 / \alpha}, \quad \alpha \in\{p, q\} .
$$

As Kristály points out in [1] , since $\Omega$ is unbounded, the loss of compactness of the Sobolev embedding $W_{0}^{1, \alpha}(\Omega) \hookrightarrow L^{\beta}(\Omega), \beta \in\left[\alpha, \alpha^{*}\right], \alpha \in\{p, q\}$, makes standard variational techniques more delicate. For this reason, we consider the subgroup $G$ of $O(l)$ defined by $G=\mathrm{id}^{m} \times O(l)$. The action of $G$ on $W_{0}^{1, \alpha}(\Omega)$ is defined by

$$
g u\left(x_{1}, x_{2}\right)=u\left(x_{1}, g_{1}^{-1} x_{2}\right)
$$

for each $\left(x_{1}, x_{2}\right) \in \omega \times \mathbb{R}^{l}, g=\mathrm{id}^{m} \times g_{1} \in G$ and $u \in W_{0}^{1, \alpha}(\Omega)$. Let

$$
W_{0, G}^{1, \alpha}(\Omega)=\operatorname{Fix} W_{0}^{1, \alpha}(\Omega)=\left\{u \in W_{0}^{1, \alpha}(\Omega): g u=u, \forall g \in G\right\} .
$$

Hence, the elements of $W_{0, G}^{1, \alpha}(\Omega)$ are the axially symmetric functions of $W_{0}^{1, \alpha}(\Omega)$.

Obviously, the action $G$ on $W_{0, G}^{1, \alpha}(\Omega)$ is isometric, that is

$$
\|g u\|_{\alpha}=\|u\|_{\alpha}, \quad \text { for all } g \in G \text {. }
$$

Since $l \geqslant 2$, the embedding $W_{0, G}^{1, \alpha}(\Omega) \hookrightarrow L^{\beta}(\Omega), \alpha<\beta<\alpha^{*}, \alpha \in\{p, q\}$, is compact [2]. In the space $W_{0}^{1, p}(\Omega) \times W_{0}^{1, q}(\Omega)$, endowed with the norm

$$
\|(u, v)\|_{p, q}=\|u\|_{p}+\|v\|_{q},
$$

one has

$$
\begin{aligned}
\operatorname{Fix}_{G}\left(W_{0}^{1, p}(\Omega) \times W_{0}^{1, q}(\Omega)\right) & =\left\{(u, v) \in W_{0}^{1, p}(\Omega) \times W_{0}^{1, q}(\Omega): g(u, v)=(u, v), \forall g \in G\right\} \\
& =W_{0, G}^{1, p}(\Omega) \times W_{0, G}^{1, q}(\Omega) .
\end{aligned}
$$

\section{Main result}

Our main result is the following.

Theorem 2.1. Let $F: \Omega \times \mathbb{R}^{2} \rightarrow \mathbb{R}$ be a continuous function that satisfies conditions $\left(1_{F}\right)-\left(4_{F}\right)$. Furthermore, assume that

(5) $\limsup _{|(\xi, \eta)| \rightarrow+\infty} \frac{F(x, \xi, \eta)}{|\xi|^{p}+|\eta|^{q}} \leqslant 0$ uniformly for every $x \in \Omega$;

(6) there exists $\left(u_{0}, v_{0}\right) \in W_{0, G}^{1, p}(\Omega) \times W_{0, G}^{1, q}(\Omega)$ such that

$$
\int_{\Omega} F\left(x, u_{0}(x), v_{0}(x)\right) \mathrm{d} x>0 .
$$

Then there exist a number $\sigma$ and a non-degenerate compact interval $C \subseteq[0,+\infty[$ such that, for every continuous function $H: \Omega \times \mathbb{R}^{2} \rightarrow \mathbb{R}$ satisfying conditions $\left(1_{H}\right)-\left(4_{H}\right)$ and for every $\lambda \in C$, there exists $\delta>0$ such that, for each $\mu \in] 0, \delta\left[\right.$, the problem $\left(S_{\lambda, \mu}\right)$ has at least two solutions, denoted by $\left(u_{\lambda, \mu}^{i}, v_{\lambda, \mu}^{i}\right), i \in\{1,2\}$, with $u_{\lambda, \mu}^{i}$ and $v_{\lambda, \mu}^{i}$ axially symmetric and with norms less than $\sigma$. 
Proof. Let $X=W_{0, G}^{1, p}(\Omega) \times W_{0, G}^{1, q}(\Omega)$. We define two functionals $\Phi$ and $\mathcal{F}$ by setting, for each $(u, v) \in X$,

$$
\begin{aligned}
\Phi(u, v) & =\frac{1}{p}\|u\|_{p}^{p}+\frac{1}{q}\|v\|_{q}^{q}, \\
\mathcal{F}(u, v) & =-\int_{\Omega} F(x, u(x), v(x)) \mathrm{d} x .
\end{aligned}
$$

In view of $\left(3_{F}\right)$ and $\left(4_{F}\right)$, and using the Sobolev embeddings, we can prove that $\mathcal{F}$ is a class- $C^{1}$ function; its differential is given by

$$
\mathcal{F}^{\prime}(u, v)(w, y)=-\int_{\Omega}\left[F_{u}(x, u, v) w+F_{v}(x, u, v) y\right] \mathrm{d} x .
$$

By the same arguments as used in the proof of $\left[\mathbf{1}\right.$, Theorem 2.2], owing to $\left(1_{F}\right),\left(3_{F}\right)$ and (6) there exists $\rho>0$ such that the functional

$$
\mathcal{G}(u, v, \lambda)=\Phi(u, v)+\lambda \mathcal{F}(u, v)+\lambda \rho
$$

satisfies the inequality

$$
\sup _{\lambda \in I} \inf _{(u, v) \in X} \mathcal{G}(u, v, \lambda)<\inf _{(u, v) \in X} \sup _{\lambda \in I} \mathcal{G}(u, v, \lambda),
$$

where $I=[0,+\infty[$. Now, we wish to apply Theorem 1.1 to the continuous functional $\mathcal{G}$. Clearly, for each $(u, v) \in X$, the functional $\mathcal{G}(u, v, \cdot)$ is concave in $I$.

Fix $\lambda \in I$. Since $W_{G}^{1, \alpha}(\Omega) \hookrightarrow L^{\alpha}(\Omega)$ is continuous, there exist two positive constants, $c_{1}$ and $c_{2}$, such that

$$
\|u\|_{L^{p}} \leqslant c_{1}\|u\|_{p} \quad \text { and } \quad\|v\|_{L^{q}} \leqslant c_{2}\|v\|_{q} .
$$

Let

$$
a<\min \left\{\frac{1}{\lambda p c_{1}^{p}}, \frac{1}{\lambda q c_{2}^{q}}\right\} .
$$

Since (5) holds, there exists a function $k_{a} \in L^{1}(\Omega)$ such that

$$
F(x, \xi, \eta) \leqslant a\left(|\xi|^{p}+|\eta|^{q}\right)+k_{a}(x)
$$

for all $(\xi, \eta) \in \mathbb{R}^{2}$ and $x \in \Omega$.

Fix $(u, v) \in X$. From the last inequality we deduce that

$$
\int_{\Omega} F(x, u(x), v(x)) \mathrm{d} x \leqslant a\left(c_{1}^{p}\|u\|_{p}^{p}+c_{2}^{q}\|v\|_{q}^{q}\right)+\left\|k_{a}\right\|_{L^{1}} .
$$

So,

$$
\mathcal{G}(u, v, \lambda) \geqslant\left(\frac{1}{p}-\lambda c_{1}^{p} a\right)\|u\|_{p}^{p}+\left(\frac{1}{q}-\lambda c_{2}^{q} a\right)\|v\|_{q}^{q}-\lambda\left\|k_{a}\right\|_{L^{1}}+\lambda \rho,
$$

i.e. $\mathcal{G}(\cdot, \cdot, \lambda)$ is coercive. 
Fix $\lambda \in I$. In view of $\left(1_{F}\right)$ and $\left(4_{F}\right)$, by [1, Lemma 3.4], the functional $\mathcal{F}$ is sequentially weakly continuous on $X$. Thus, the functional $\mathcal{G}(\cdot, \cdot, \lambda)$ is sequentially weakly lower semicontinuous in $X$.

Now, fixing $\gamma>\sup _{\lambda \in I} \inf _{(u, v) \in X} \mathcal{G}(u, v, \lambda)$, Theorem 1.1 ensures that there exists a non-empty open set $A \subseteq I$ with the following property: for every $\lambda \in A$ and every continuous function $H: \Omega \times \mathbb{R}^{2} \rightarrow \mathbb{R}$ satisfying conditions $\left(1_{H}\right)-\left(4_{H}\right)$, there exists $\delta>0$ such that, for each $\mu \in] 0, \delta[$, the functional

$$
E_{\lambda, \mu}(u, v)=\mathcal{G}(u, v, \lambda)+\mu \mathcal{H}(u, v)
$$

has at least two local minima lying in the set $\{(u, v) \in X: \mathcal{G}(u, v, \lambda)<\gamma\}$, namely $\left(u_{\lambda, \mu}^{i}, v_{\lambda, \mu}^{i}\right), i \in\{1,2\}$, where $\mathcal{H}$ is the sequentially weakly continuous functional defined by

$$
\mathcal{H}(u, v)=-\int_{\Omega} H(x, u(x), v(x)) \mathrm{d} x .
$$

Since $F$ and $H$ are axially symmetric in the first variable, and each $g \in G$ is isometric, the function $E_{\lambda, \mu}$ is $G$-invariant, i.e.

$$
E_{\lambda, \mu}(g(u, v))=E_{\lambda, \mu}(g u, g v)=E_{\lambda, \mu}(u, v)
$$

for each $g \in G,(u, v) \in W_{0}^{1, p}(\Omega) \times W_{0}^{1, q}(\Omega)$. As

$$
\operatorname{Fix}\left(W_{0}^{1, p}(\Omega) \times W_{0}^{1, q}(\Omega)\right)=W_{0, G}^{1, p}(\Omega) \times W_{0, G}^{1, q}(\Omega),
$$

by the principle of symmetric criticality of [3], we find that $\left(u_{\lambda, \mu}^{i}, v_{\lambda, \mu}^{i}\right), i \in\{1,2\}$, are also the critical points of $E_{\lambda, \mu}$ and then weak solutions of the problem $\left(S_{\lambda, \mu}\right)$.

Finally, let $[a, b] \subset A$ be any non-degenerate compact interval. Observe that

$$
\begin{aligned}
& \bigcup_{\lambda \in[a, b]}\{(u, v) \in X: \mathcal{G}(u, v, \lambda) \leqslant \gamma\} \\
& \subseteq\{(u, v) \in X: \mathcal{G}(u, v, a) \leqslant \gamma\} \cup\{(u, v) \in X: \mathcal{G}(u, v, b) \leqslant \gamma\} .
\end{aligned}
$$

This implies that the set

$$
S:=\bigcup_{\lambda \in[a, b]}\{(u, v) \in X: \mathcal{G}(u, v, \lambda) \leqslant \gamma\}
$$

is bounded. Hence, the local minima of $E_{\lambda, \mu}$ have norm less than or equal to $\sigma$, taking $\sigma=\sup _{(u, v) \in S}\|(u, v)\|_{p, q}$. This concludes the proof.

Now, we give an example in which the hypotheses of Theorem 2.1 are satisfied.

Example 2.2. Let $\Omega=\omega \times \mathbb{R}^{2}$, where $\omega$ is a bounded open interval in $\mathbb{R}$. Let $\alpha, \beta$ : $\Omega \rightarrow \mathbb{R}$ be two continuous, non-negative, not identically zero, axially symmetric functions with compact support in $\Omega$. Then there exist a number $\sigma$ and a non-degenerate compact 
interval $C \subseteq\left[0,+\infty[\right.$ such that, for every $a \in] \frac{3}{2}, 3[, b \in] \frac{9}{4}, 9[, \lambda \in C$, there exists $\delta>0$ such that, for each $\mu \in] 0, \delta[$, the system

$$
\begin{aligned}
-\Delta_{3 / 2} u & =\frac{5}{2} \lambda \alpha(x)|u|^{1 / 2} u \cos \left(|u|^{5 / 2}+|v|^{3}\right)+\mu \beta(x) a u|u|^{a-2} & & \text { in } \Omega, \\
-\Delta_{9 / 4} v & =3 \lambda \alpha(x)|v| v \cos \left(|u|^{5 / 2}+|v|^{3}\right)+\mu \beta(x) b v|v|^{b-2} & & \text { in } \Omega, \\
u & =v=0 & & \text { on } \partial \Omega,
\end{aligned}
$$

has at least two solutions with the properties from Theorem 2.1.

In this case we have

$$
F(x, \xi, \eta)=\alpha(x) \sin \left(|\xi|^{5 / 2}+|\eta|^{3}\right) \quad \text { and } \quad H(x, \xi, \eta)=\beta(x)\left(|\xi|^{a}+|\eta|^{b}\right)
$$

for each $(x, \xi, \eta) \in \Omega$. It is easy to observe that conditions $\left(1_{F}\right)-\left(3_{F}\right)$ and $\left(1_{H}\right)-\left(3_{H}\right)$ hold immediately, while $\left(4_{F}\right)$ is verified by choosing $r=\frac{11}{4}, s=\frac{33}{8}$, and $\left(4_{H}\right)$ is verified choosing $r \in] a, 3[, s \in] b, 9$ [ with $s=\frac{3}{2} r$. Finally, (5) is obvious and (6) follows by putting $u_{0}(x)=\left(\frac{1}{2} \pi\right)^{2 / 5}$ for every $x \in \operatorname{supp} \alpha$ and $v_{0}(x)=0$ for every $x \in \Omega$.

By the same arguments as used in the proof of Theorem 2.1, but applying also the Palais-Smale properties, we obtain the result below. We recall that a Gâteaux differentiable functional $S$ on a real Banach space $X$ is said to satisfy the Palais-Smale condition if each sequence $\left\{x_{n}\right\}$ in $X$ such that $\sup _{n \in \mathbb{N}}\left|S\left(x_{n}\right)\right|<+\infty$ and $\lim _{n \rightarrow+\infty}\left\|S^{\prime}\left(x_{n}\right)\right\|_{X}=0$ admits a strongly converging subsequence.

Theorem 2.3. Assume that the hypotheses of Theorem 2.1 hold.

Then there exists a non-empty open set $A \subseteq[0,+\infty[$ such that, for every $\lambda \in A$ and for every continuous function $H: \Omega \times \mathbb{R}^{2} \rightarrow \mathbb{R}$ satisfying conditions $\left(1_{H}\right)-\left(4_{H}\right)$ and

$\left(5_{H}\right) \limsup _{|(\xi, \eta)| \rightarrow+\infty} \frac{H(x, \xi, \eta)}{|\xi|^{p}+|\eta|^{q}}<+\infty$ uniformly for every $x \in \Omega$,

there exists $\delta>0$ such that, for each $\mu \in] 0, \delta\left[\right.$, the problem $\left(S_{\lambda, \mu}\right)$ has at least three solutions axially symmetric.

Proof. Let $A$ and $E_{\lambda, \mu}$ have the same meaning as in the proof of Theorem 2.1, $H$ : $\Omega \times \mathbb{R}^{2} \rightarrow \mathbb{R}$ being a continuous function satisfying $\left(5_{H}\right)$. Reasoning as in the proof of Theorem 2.1, there exists $\delta_{1}>0$ such that, for each $\left.\mu \in\right] 0, \delta_{1}\left[\right.$, the problem $\left(S_{\lambda, \mu}\right)$ has at least two solutions.

First of all, the functional $E_{\lambda, \mu}$ is coercive. In fact, from $\left(5_{H}\right)$, there exist a positive constant $b \in \mathbb{R}$ and a function $k_{b}(x) \in L^{1}(\Omega)$ such that

$$
H(x, \xi, \eta) \leqslant b\left(|\xi|^{p}+|\eta|^{q}\right)+k_{b}(x)
$$

for all $x \in \Omega$ and $(\xi, \eta) \in \mathbb{R}^{2}$.

Fix $(u, v) \in X$. From the previous inequality we deduce that

$$
\mathcal{H}(u, v)=-\int_{\Omega} H(x, u(x), v(x)) \mathrm{d} x \geqslant-b\left(c_{1}^{p}\|u\|_{p}^{p}+c_{2}^{q}\|v\|_{q}^{q}\right)-\left\|k_{b}\right\|_{L^{1}} .
$$


Let

$$
\delta<\min \left\{\delta_{1}, \frac{1}{b}\left(\frac{1}{p c_{1}^{p}}-\lambda a\right), \frac{1}{b}\left(\frac{1}{q c_{2}^{q}}-\lambda a\right)\right\} .
$$

So, for each $\lambda \in A$ and $\mu \in] 0, \delta[$, we have

$$
\begin{aligned}
E_{\lambda, \mu} & (u, v) \\
& =\mathcal{G}(u, v, \lambda)+\mu \mathcal{H}(u, v) \\
& \geqslant\left(\frac{1}{p}-c_{1}^{p}(\lambda a+\mu b)\right)\|u\|_{p}^{p}+\left(\frac{1}{q}-c_{2}^{q}(\lambda a+\mu b)\right)\|v\|_{q}^{q}-\lambda\left\|k_{a}\right\|_{L^{1}}+\lambda \rho-\mu\left\|k_{b}\right\|_{L^{1}}
\end{aligned}
$$

for all $(u, v) \in X$. This ensures the coercivity of the functional $E_{\lambda, \mu}$ for each $\lambda \in A$ and $\mu \in] 0, \delta[$.

Now, let us check the Palais-Smale condition for $E_{\lambda, \mu}$. To this end, let $\left\{\left(u_{n}, v_{n}\right)\right\}$ be a sequence in $X$ satisfying

$$
\sup _{n \in \mathbb{N}}\left|E_{\lambda, \mu}\left(u_{n}, v_{n}\right)\right| \leqslant M, \quad \lim _{n \rightarrow \infty}\left\|E_{\lambda, \mu}^{\prime}\left(u_{n}, v_{n}\right)\right\|_{X^{*}}=0 .
$$

Since the functional $E_{\lambda, \mu}$ is coercive, the sequence $\left\{\left(u_{n}, v_{n}\right)\right\}$ is bounded in $X$. So, applying $\left[\mathbf{1}\right.$, Lemma 3.5] to the functional $E_{\lambda, \mu}(\cdot, \cdot)$ we obtain that $\left\{\left(u_{n}, v_{n}\right)\right\}$ contains a strongly convergent subsequence in $X$.

Since the functional $E_{\lambda, \mu}$ is $C^{1}$ in $X$, our conclusion follows by [4, Corollary 1], which ensures that there exists a third critical point of the functional $E_{\lambda, \mu}$ which is a solution of problem $\left(S_{\lambda, \mu}\right)$.

\section{References}

1. A. KRISTÁLy, Existence of two nontrivial solutions for a class of quasilinear elliptic variational systems on strip-like domains, Proc. Edinb. Math. Soc. 48 (2005), 465-477.

2. P. L. Lions, Symétrie et compacité dans les espaces de Sobolev, J. Funct. Analysis 49 (1982), 315-334.

3. R. S. Palais, The principle of symmetric criticality, Commun. Math. Phys. 69 (1979), 19-30.

4. P. PuCCi ANd J. Serrin, A mountain pass theorem, J. Diff. Eqns 60 (1985), 142-149.

5. B. RICCERI, On a three critical points theorem, Arch. Math. 75 (2000), 220-226.

6. B. RICCERI, Minimax theorems for limits of parametrized functions having at most one local minimum lying in a certain set, Topol. Applic. 153 (2006), 3308-3312. 